\title{
Alternative Vinen's equation and its extension to rotating counterflow superfluid turbulence
}

\author{
M. Sciacca ${ }^{1}$, M.S. Mongiovi ${ }^{1 *}$ and D. Jou ${ }^{2}$ \\ ${ }^{1}$ Dipartimento di Metodi e Modelli Matematici Università di Palermo, c/o Facoltà di Ingegneria, \\ Viale delle Scienze, 90128 Palermo, Italy \\ ${ }^{2}$ Departament de Física, Universitat Autònoma de Barcelona, 08193 Bellaterra, Catalonia, Spain
}

Key words: superfluid turbulence; vortex tangle; rotating counterflow turbulence PACS number(s): 67.40.Vs, 67.40.Bz, 47.27.2i, 05.70.Ln

\begin{abstract}
Two alternative Vinen's evolution equations for the vortex line density $L$ in counterflow superfluid turbulence, are physically admissible and lead to analogous results in steady states. In Phys. Rev. B, 69, 094513 (2004) the most used of them was generalized to counterflow superfluid turbulence in rotating containers. Here, the analogous generalization for the alternative Vinen's equation is proposed. Both generalized Vinen's equations are compared with the experimental results, not only in steady-states but also in some unsteady situations. From this analysis follows that the solutions of the alternative Vinen's equation tend significantly faster to the corresponding final steady state values than the solutions of the usual Vinen's equation, and that the latter seems more suitable for the description of the experimental available data.
\end{abstract}

\section{Introduction}

There is experimental evidence that turbulent helium II has a peculiar behavior due to quantum effects at some length scales, while at other length scales it appears similar to classical hydrodynamic turbulence [1]-[5]. Quantum turbulence is described as a chaotic motion of quantized vortices in a disordered tangle. The measurements of vortex lines are described in terms of a macroscopic average of the vortex line length per unit volume $L$ (briefly called vortex line density and which has dimensions length $h^{-2}$ ).

The evolution equation for $L$ under constant values of the counterflow velocity $\mathbf{V}(\mathbf{V}=<$ $\mathbf{v}_{\mathbf{n}}-\mathbf{v}_{\mathbf{s}}>, \mathbf{v}_{n}$ and $\mathbf{v}_{s}$ being the velocities of the normal and superfluid components) and in absence of rotation was formulated by Vinen. Neglecting the influence of the walls, such an equation is [6]:

$$
\frac{d L}{d t}=\alpha V L^{3 / 2}-\beta \kappa L^{2}
$$

\footnotetext{
${ }^{*}$ Corresponding author.

${ }^{0}$ E-mail addresses: msciacca@unipa.it (M. Sciacca), mongiovi@unipa.it (M. S. Mongiovì), david.jou@uab.es (D. Jou)
} 
with $V=|\mathbf{V}|, \kappa=h / m$ the quantum of vorticity ( $m$ the mass of the ${ }^{4}$ He atom and $h$ Planck's constant, $\kappa \simeq 9.9710^{-4} \mathrm{~cm}^{2} / \mathrm{s}$ ) and $\alpha$ and $\beta$ dimensionless parameters.

Note however that another version of (1.1) is the so-called alternative Vinen's equation, which is also admissible on dimensional grounds [7]-[9]:

$$
\frac{d L}{d t}=A_{1} \frac{V^{2}}{\kappa} L-\beta \kappa L^{2} .
$$

The steady state solutions of (1.1) and (1.2) are $L=(\alpha V / \beta \kappa)^{2}$ and $L=A_{1} V^{2} / \beta \kappa^{2}$ respectively, in agreement with the experimental results in completely developed turbulent regime, which lead to $L^{1 / 2}=\gamma V / \kappa$, with $\gamma$ a dimensionless coefficient which depends on the temperature. Therefore, the difference between (1.1) and (1.2) must be searched in the dynamical aspects. This was carried out by Vinen himself (see sections 6 and 7 of Ref. 7]) and in more detail by Nemirovskii et al. [9] without arriving to definite conclusions, because the predictions of (1.1) and (1.2) in the domain of available experimental results are very similar to each other.

Here we will look for a more general situation where the difference between (1.1) and (1.2) becomes enhanced. Essentially, equations (1.1) and (1.2) may be obtained from a microscopic approach based on vortex dynamics, though the derivation of the first one is more direct and straightforward than the other. Before proceeding, let us briefly recall that from a microscopic approach based on vortex dynamics the production term may be shown to be proportional to $V L / R_{\text {peak }}$, where $R_{\text {peak }}$ is the intervortex spacing [8, 10]. Since the latter is of the form $L^{-1 / 2}$, the form $V L^{3 / 2}$ adopted in (1.1) follows in an immediate way. However, it has been also argued that [8], since in the steady state $L^{1 / 2}$ is proportional to $V$, one could also write $R_{\text {peak }}$ as inversely proportional to $V$, in which case one would have for the production term the alternative form $V^{2} L$ adopted in (1.2). The equation (1.2) was also derived by Lipniacki (pag. 177 of Refs. [2, 11]) through an alternative microscopic approach, which is referred to the reconnections of vortex lines.

Let us say, in support of the form $V^{2} L$, that though it is less natural from a kinematical point of view, it is rather natural from an energetic point of view, if one assumes in agreement with the microscopical model ${ }^{1,2}$ that the friction force between the normal fluid and the vortex tangle is proportional to $V L$. In this case, the power delivered to the tangle per unit volume would be proportional to $V^{2} L$. Since the energy density of the tangle is proportional to $L$, the production contribution to $d L / d t$ would be proportional to the power delivered to the tangle, i.e. to $V^{2} L$.

Another motivation in support of (1.2) is that it is closer to the phenomenological theory of classical turbulence [12] than equation (1.1). Indeed, by assuming that turbulence can be characterized by the line density $L$ and that its derivative $d L / d t$ is an analytic function of $L$, the relation $A_{1} V^{2} L$ can be interpreted as the first term in a series expansion. However, equation (1.1) has been much more used than equation (1.2). Both (1.1) and (1.2) are particular cases of:

$$
\frac{d L}{d t}=A_{n} \kappa L^{2}\left(\frac{V}{\kappa L^{1 / 2}}\right)^{n}-\beta \kappa L^{2},
$$

in fact for $n=1$ one obtains (1.1), for $n=2$ one has (1.2), and the cases $1 \leq n \leq 2$ could correspond to fractal (intermittent) turbulence [13]-[15].

In recent years there has been growing attention in superfluid turbulence in rotating containers [16]-[18], in which the formation of vortex lines is due both to the counterflow and 
the rotation, which has fostered the extension of Vinen's ideas to a wider range of situations [15, 19, 20]. In Ref. [19], a phenomenological generalization of Vinen's equation (1.1) has been proposed for the evolution of $L$ in the simultaneous presence of $\mathbf{V}$ and $\boldsymbol{\Omega}$ ( $\boldsymbol{\Omega}$ being the angular velocity of the container). A thermodynamic analysis to determine possible coupling terms between the evolution equations of $L$ and $\mathbf{V}$ has been performed in Refs. [15].

Here, the extension of the form (1.2) of Vinen's equation to rotating counterflow turbulence will be studied in order to explore whether this more general situation may provide further arguments to decide which of both starting equations, (1.1) or (1.2), is more suitable to describe actual experimental results. Of course, the final version will be fully satisfactory only when the macroscopic expression will be understood from a microscopical basis, and the respective coefficients of all terms will be microscopically calculated and found to coincide with macroscopic observations. However, this situation is still far ahead of our present abilities, because of the difficulties in modeling — in a statistically significative way - a system of vortices under rotation. Thus, a combined effort in macroscopic and microscopic perspectives seems a reasonable and promising way to proceed.

The plan of the paper is the following. In Section 2 a new equation for the evolution of $L$ in counterflow in rotating containers is written, through a modification of the Vinen's alternative equation, and it is solved in steady and unsteady situations in order to compare it with the generalization of the usual Vinen's one made in Ref. [19. In Section 3 a thermodynamic analysis of counterflow rotational superfluid turbulence is performed, according to the formalism of nonequilibrium thermodynamics, to obtain the general form of the friction exerted by the tangle on the motion of the fluid.

\section{New equation for the dynamics of $L$ in rotating counterflow superfluid turbulence}

There are not many experiments on counterflow in rotating containers. In the work of Swanson et al. [22, the counterflow velocity $V$ was parallel to the rotation axis and the experimental observations consisted in measuring the attenuation of second sound, when it is propagated orthogonal to the rotation axis. They interpreted their results as measurements of the vortex line density $L$, and compared the observed line density with what would be expected if the two sources of vorticity (rotation and counterflow) simply added. Their results showed an interesting interplay between the ordered vortices of rotation and the disordered ones of counterflow. They found a critical counterflow velocity which marks the transition to a disordered turbulent tangle. In the limit of high rotation this critical velocity appears proportional to $\sqrt{\Omega}$.

Another experiment is that carried out by Yarmchuck and Glaberson [23, in which a pair of horizontal parallel glass plates are arranged to form a large aspect ratio channel closed at one end with a heater nearby, and open at the other end to the liquid helium bath. The channel is rotated about a vertical axis orthogonal to the heat flux. In this way the counterflow velocity $\mathbf{V}$ is orthogonal to angular velocity $\boldsymbol{\Omega}$. By determining gradient of temperature and chemical potential as functions of $V$ and $\Omega$, they found a linear regime, in which these gradients grow proportionally to the rotation speed, and a critical counterflow velocity associated with the onset of turbulent regime, which increases as $\sqrt{\Omega}$ when $\Omega$ gets large. 


\subsection{The evolution equation}

The mentioned experiments by Swanson, Barenghi and Donnelly [22] and by Yarmchuck and Glaberson 23] show that, when the heat flux and the rotation are simultaneously present, there appears a complex interaction between both processes in the formation and destruction of vortices. In the experiment performed in Ref. [22] ( $\boldsymbol{\Omega}$ and $\mathbf{V}$ collinear), they observed that the effects of $V$ and $\Omega$ are not additive: in fact, for low values of $\Omega$, the laminar regime (vortex-free regime) is absent and the total vortex line density is higher than $L_{R}+L_{H}, L_{R}$ and $L_{H}$ being the values of $L$ in steady rotation and in steady counterflow superfluid turbulence respectively:

$$
L=L_{R}=\frac{2 \Omega}{\kappa}, \quad L_{H}=\gamma^{2} \frac{V^{2}}{\kappa^{2}},
$$

with $\gamma$ a dimensionless coefficient while, for higher values of $\Omega$ and $V$, the measured values of $L$ are always less than $L_{H}+L_{R}$, and the deviation increases with $V$ and $\Omega$. Therefore, the rotation facilitates the vortex formation, in the absence or for small counterflow velocities, but it hinders their lengthening for high values of $V$ and $\Omega$.

For fast enough rotation, there are two critical counterflow-rotation velocities $V_{c 1}$ and $V_{c 2}$, which scale as $\Omega^{1 / 2}\left(V_{c 1}=C_{1} \sqrt{\Omega}, V_{c 2}=C_{2} \sqrt{\Omega}\right.$, with $C_{1}=0.053 \mathrm{~cm} \mathrm{sec}^{-1 / 2}, C_{2}=0.118$ $\left.\mathrm{cm} \mathrm{sec}^{-1 / 2}\right)$. For $V \leq V_{c 1}$, the length $L$ per unit volume of the vortex lines is independent of $V$ and agrees with the first expression in (2.1). For $V_{c 1} \leq V \leq V_{c 2}, L$ is still independent of $V$ and proportional to $\Omega$, with a slightly different proportionality constant than in the previous situation; finally, for $V \geq V_{c 2}, L$ increases and becomes proportional to $V^{2}$ at high values of $V$.

Swanson et al. [22] interpreted the first transition as the Donnelly-Glaberson instability CCD,OG: excitation of helical waves (Kelvin waves) by the counterflow on the vortex lines induced by rotation, and the second as a transition to a turbulent disordered tangle. Tsubota et al. [18] also have paid attention to this experiment. They proposed that the regime $V_{c 1}<$ $V<V_{c 2}$ is a state of polarized turbulence, while for $V>V_{c 2}$ the polarization is decreased by the large number of reconnections.

In the regime of high rotation $\left(0.2 \mathrm{~Hz} \leq \Omega / 2 \pi \leq 1.0 \mathrm{~Hz}\right.$ and $\left.0 \leq V^{2} \leq 0.2 \mathrm{~cm}^{2} / \mathrm{s}^{2}\right)$ and $\boldsymbol{\Omega}$ parallel to $\mathbf{V}$, equation (1.1) has been generalized to incorporate the presence of rotation, as [19]:

$$
\frac{d L}{d t}=-\beta \kappa L^{2}+\alpha_{1}\left[L^{1 / 2}-m_{1} \frac{\sqrt{\Omega}}{\sqrt{\kappa}}\right] V L+\beta_{2}\left[L^{1 / 2}-m_{2} \frac{\sqrt{\Omega}}{\sqrt{\kappa}}\right] \sqrt{\kappa \Omega} L
$$

where $m_{1}$ and $m_{2}$ are linked to the coefficients introduced in Ref. [19] by the relations $m_{1}=$ $\beta_{4} / \alpha_{1}$ and $m_{2}=\beta_{1} / \beta_{2}$, with the coefficients $\beta, \alpha_{1}, \beta_{1}, \beta_{2}$ and $\beta_{4}$ depending on the polarization of the tangle, which was supposed function of $\Omega$ and $V$.

Note that, as production terms in Eq. (2.2) a term in $V$ and a term in $\Omega^{1 / 2}$ were used; this was motivated by the dependence of the steady-state values of $L^{1 / 2}$, in counterflow only and in rotation only, on $V$ and on $\Omega^{1 / 2}$ (see equations (2.1)), and by the observation that the microscopic mechanism responsible for the growth of vortices (the mutual friction force) is the same in rotating helium II and in superfluid turbulence. There are present three destruction contributions: a term $-\beta \kappa L^{2}$ independent on $V$ and on $\Omega$, present also in (1.1) and (1.2) (this term, responsible for the vortex decay in pure counterflow, was determined by Vinen in analogy with classical turbulence) and the two terms $-\beta_{1} \Omega L$ and $-\beta_{4} \frac{V \sqrt{\Omega}}{\sqrt{\kappa}} L$, which take account of 
the interactions between counterflow and rotation, reducing the length of the vortices; a term quadratic in $V$ was neglected, because the values of $V$ used in the experiments by Swanson et al. were not very high.

The Eq. (2.2) describes, in good agreement with experimental results, some of the most relevant effects observed in the experiments of Ref. 22]. However, as we have mentioned in the Introduction, the alternative Vinen's equation also describes well the experimental results in pure counterflow and therefore it is natural to ask how does it work when extended to incorporate rotation.

Here, we suggest a new evolution equation for the evolution of vortex line density $L$ in rotating counterflow, starting from the alternative Vinen's equation and following the lines of thought outlined in Ref. [19]. We consider the case in which $V$ and $\Omega$ are parallel to each other. The proposed new equation, reducing to (1.2) for vanishing rotation, is:

$$
\frac{d L}{d t}=-\beta \kappa L^{2}+A_{1}\left[L-\nu_{1} \frac{\Omega}{\kappa}\right] \frac{V^{2}}{\kappa}+B_{1}\left[L-\nu_{2} \frac{\Omega}{\kappa}\right] \Omega,
$$

where the coefficients $\beta, A_{1}, \nu_{1}, B_{1}$ and $\nu_{2}$ depend on the polarization of the tangle, which was supposed function of $\Omega$ and $V$.

We outline now a possible physical interpretation for the terms of production and destruction of vortices introduced in this equation. The two production terms, $A_{1} \frac{V^{2}}{\kappa} L$ and $B_{1} \Omega L$, indicate that both rotation and counterflow favor the vortex formation: the quantities $t_{H}=\kappa /\left(A_{1} V^{2}\right)$ and $t_{R}=1 /\left(B_{1} \Omega\right)$ can be interpreted as the characteristic times for the formation of vortex lines due to the counterflow and to the rotation, respectively. As in (2.2), three destruction terms are present. The term $A_{1} \nu_{1} \Omega V^{2} / \kappa^{2}$ describes the complex interaction between rotation - which tends to straighten out the otherwise irregular vortex lines of the tangle, thus shortening them and reducing $L$ - and counterflow, which randomize them. Another aspect especially worth of comment is the meaning of the two destruction terms, independent on $V,-B_{1} \nu_{2} \Omega^{2} / \kappa$ and $-\beta \kappa L^{2}$. One could argue, indeed, that at steady pure rotation there is no vortex destruction. Thus, in purely rotation situations the vortices are usually produced on the walls and they migrate to the bulk of the fluid in the cylinder; in this case, these terms would represent a repulsion force between parallel vortices, putting an upper limit to the possible number of straight vortices in the vortex array.

In a general situation, these two destruction terms will incorporate real destruction of vortices due to breaking recombination of nonparallel vortices, and to repulsion between parallel segments of vortices in the presence of rotation. The superposition of these two different effects is one of the reasons that the coefficients in the terms in $L^{2}$ and in $\Omega^{2}$ depend on the polarization of the tangle. The destruction term $-\beta \kappa L^{2}$, which appears also in (1.1), (1.2) and (2.2), is not modified, in agreement with recent studies which show that the decay of the turbulence, in the absence of rotation and counterflow velocity, is analogous to that of classical turbulence [12.

As it was shown in Ref. [19], the simplicity of (2.2) as (2.3) is a little bit deceptive, because the coefficients appearing in it depend on the polarity and the anisotropy of the vortex tangle, which are taken as independent variables in the more detailed approach proposed in the recent paper [13]. Of course, this difficulty arises not only in the macroscopic approach, but also in the microscopic approaches. Since the coefficients appearing in (2.3) depend on the anisotropy and on the polarization of the tangle, they have to depend on the angular velocity $\Omega$ and on the counterflow velocity $V$. In particular, when $\Omega=0$ Eq. (2.3) reduces to (1.2), and the 
coefficient $A_{1} / \beta$ assumes the value:

$$
\frac{A_{1}}{\beta}=0.156 \text {. }
$$

In the analysis carried out in the present paper, the dependence of the coefficients on the polarity plays not an important role, because in Section 2.2 we are comparing two equations in a regime of values of $V$ and $\Omega\left(0.2 \mathrm{~Hz} \leq \Omega / 2 \pi \leq 1.0 \mathrm{~Hz}\right.$ and $\left.0 \leq V^{2} \leq 0.2 \mathrm{~cm}^{2} / \mathrm{s}^{2}\right)$ with approximately the same polarity, and in Section 2.4 we compare the non-stationary behavior of the perturbations to a given physical situation, as described by two different equations.

In the successive subsections we solve equation (2.3) in steady and unsteady situations and we show that it allows us to account for the experiments described in Ref. [22] and we compare the results with the description pointed in Ref. [19] based on the extension of (1.1).

\subsection{The stationary solutions and their stability}

The non zero stationary solutions of (2.3) are solutions of the following second-order algebraic equation in the unknown $L$ :

$$
\beta \kappa L^{2}-\left[\frac{A_{1}}{\kappa} V^{2}+B_{1} \Omega\right] L+\left[B_{2} \frac{\Omega^{2}}{\kappa}+\frac{B_{3}}{\kappa^{2}} V^{2} \Omega\right]=0,
$$

where we have put $B_{2}=B_{1} \nu_{2}$ and $B_{3}=A_{1} \nu_{1}$.

Looking at the experimental results of Ref. [22], one notes that $L$ is almost independent of $V$ for $V<V_{c 2}$, with a step change around $V_{c 1}$, while there is a variation of the slope near $V_{c 2}$. We will concentrate on the change near $V_{c 2}$. Reasoning as in Ref. [19], we observe that, under the hypothesis:

$$
\frac{B_{2}}{\beta}=\frac{B_{3}}{A_{1}}\left(\frac{B_{1}}{\beta}-\frac{B_{3}}{A_{1}}\right)
$$

it follows that:

$$
\frac{B_{1}}{\beta}=\frac{\nu_{1}^{2}}{\nu_{1}-\nu_{2}}, \quad \frac{B_{2}}{\beta}=\frac{\nu_{1}^{2} \nu_{2}}{\nu_{1}-\nu_{2}}
$$

and the solutions of equation (2.5) can be written:

$$
\begin{gathered}
L=L_{1}^{A}=\nu_{1} \frac{\Omega}{\kappa}, \\
L=L_{2}^{A}=\frac{A_{1}}{\beta} \frac{V^{2}}{\kappa^{2}}+\left(\frac{B_{1}}{\beta}-\nu_{1}\right) \frac{\Omega}{\kappa} .
\end{gathered}
$$

In the plane $\left(V^{2}, L\right),(2.8)$ and (2.9) represent two families of straight lines plotted in Fig. 1, the first of them (equation (2.8)) parallel to the $V^{2}$ axis and the second one (equation (2.9)) with a slope independent of $\Omega$. A linear stability analysis of these solutions shows that the solution (2.8) is stable if $V$ is lower than:

$$
V_{c 2}^{2}=\frac{\beta}{A_{1}}\left[2 \frac{B_{3}}{A_{1}}-\frac{B_{1}}{\beta}\right] \Omega \kappa=\frac{\beta}{A_{1}} \frac{\nu_{1}^{2}-2 \nu_{1} \nu_{2}}{\nu_{1}-\nu_{2}} \Omega \kappa,
$$

(corresponding to the point of interception of the two straight lines (2.8) and (2.9)), while, for values of $V$ higher than $V_{c 2}$, the solution (2.9) is stable. Therefore $V_{c 2}$ represents the second 
critical counterflow-rotation velocity observed in the experiments of Ref. [22]. As we see, this critical velocity scales as $\sqrt{\Omega}$, in agreement with experimental observations.

The experimental data on the steady states of $L$ allow us to determine the values assumed by the dimensionless quantities appearing in equation (2.3). One obtains:

$$
\frac{A_{1}}{\beta}=0.0125, \quad \frac{B_{1}}{\beta}=3.90, \quad \frac{B_{2}}{\beta}=3.79, \quad \frac{B_{3}}{\beta}=0.025,
$$

from which we obtain:

$$
\nu_{1}=2.036, \quad \nu_{2}=0.97 \text {. }
$$

The coefficient $\beta$, which controls the rate of evolution of $L$, cannot be determined from the knowledge of the stationary solutions. Comparing the value $A_{1} / \beta$ obtained in the combined situation with the value (2.4) obtained in absence of rotation, we can deduce that the first one is approximately 12 times the second one, which means that the coefficient $A_{1} / \beta$ depends on the anisotropy and the polarity of the tangle, which depend on $\Omega$ and $V$.

Using the obtained values of the dimensionless quantities (2.11) and (2.12), the steady stationary solutions $L_{1}^{A}$ and $L_{2}^{A}$ become:

$$
L_{1}^{A}=2.036 \frac{\Omega}{\kappa}, \quad \text { and } \quad L_{2}^{A}=0.0125 \frac{V^{2}}{\kappa^{2}}+1.86 \frac{\Omega}{\kappa} .
$$

In Fig. 1 a comparison of such stationary solutions $L_{1}^{A}$ and $L_{2}^{A}$ with the experimental data of Swanson et al. is shown [22]. The conclusion of such a fit is that the stationary vortex line density $L_{1}^{A}$ and $L_{2}^{A}$, solutions of the alternative Vinen's equation in the combined situation, are in good agreement with experimental data of Swanson et al.

In Ref. [19], the stationary solutions of equation (2.2) had the form:

$$
L_{1}^{1 / 2}=1.427 \sqrt{\frac{\Omega}{\kappa}} \quad \text { and } \quad L_{2}^{1 / 2}=0.047 \frac{V}{\kappa}+1.25 \sqrt{\frac{\Omega}{\kappa}}
$$

and the comparison with the experimental data led also to the conclusion that (2.14) agree with the experiments by Swanson et al. [22]. Through (2.13) and (2.14) have a different mathematical form, in the range of the available experimental data, both of them lead to reasonable results.

From such conclusions an interesting problem is to establish which equation, either (2.2) based on the usual Vinen's equation or (2.3) based on the alternative Vinen's equation, fits better the experimental data obtained by Swanson, Donnelly and Barenghi [22].

From a first comparison, the two stationary solutions $(2.14 \mathrm{a})$ and $(2.13 \mathrm{a})$ represent the same straight line in the plane $\left(L, V^{2}\right)$ in the range $V_{c 1}^{2}<V^{2}<V_{c 2}^{2}$. So, an eventual difference between both equations could be found in the range $V^{2}>V_{c 2}^{2}$. To do that, we calculate the errors $\sigma$ between $L_{2}$ and the corresponding experimental value $\bar{L}$, and $\sigma^{A}$ between $L_{2}^{A}$ and $\bar{L}$, respectively, in such a way that we can compare the accuracy of the two models.

To find these errors, we consider the experimental values $V_{i}^{2}$ and $\Omega_{j}$ of the experiments to which $L_{2 i j}, L_{2 i j}^{A}$ and $\bar{L}_{i j}$ correspond, obtaining:

$$
\sigma=\sqrt{\frac{\sum_{i, j}\left(L_{2 i j}-\bar{L}_{i j}\right)^{2}}{N}}=963
$$


and

$$
\sigma^{A}=\sqrt{\frac{\sum_{i, j}\left(L_{2 i j}^{A}-\bar{L}_{i j}\right)^{2}}{N}}=419,
$$

where $N$ is the number of experimental data, which is equal for both cases. From (2.15) and (2.16) we can establish that the stationary solution of the alternative Vinen's equation approaches better the experimental data (for $V^{2}>V_{c 2}^{2}$ ) than that of the usual Vinen's equation.

\subsection{The first critical velocity}

The model based on the equation (2.3) does not describe the existence of the first critical velocity $V_{c 1}$ mentioned in Section (2.1), in which the value of $L$ has a small steep change. To do this, we assume that the coefficient $\nu_{1}$ depends on $\Omega$ and $V$ as:

$$
\nu_{1}=A\left\{1-B \tanh \left[N^{\prime}\left(\frac{k \Omega}{V^{2}}-C\right)\right]\right\}
$$

with $A, B$ and $C$ constants. Thus, for $V^{2} \ll V_{c 1}^{2}=\frac{1}{C} k \Omega$, it results $\nu_{1} \simeq A-B A$ and for $V^{2} \gg V_{c 1}^{2}, \nu_{1}=A+B A$, while the constant $C$ is related to $V_{c 1}$, and $2 B$ gives the size of the step of $\nu_{1}$ near $V_{c 1}$. In fact, if $V_{c 1}$ is small, the domain of $V$ in which the mentioned transition occurs is very narrow, as observed in experiments.

In (2.17), the critical value $V_{c 1}$ of the counterflow velocity is given in terms of coefficient $C$ by:

$$
V_{c 1}^{2}=\frac{1}{C} \kappa \Omega
$$

Using the experimental values of $V_{c 1}\left(V_{c 1}=0.053 \sqrt{\Omega} \mathrm{cm} \mathrm{sec}^{-1 / 2}\right)$, it is seen that $C=$ $\kappa /(0.053)^{2}=0.355$.

To determine the coefficients $A$ and $B$ in (2.17), we consider that for a given value of $\Omega$, for small values of $V$, the tangle will be completely oriented along the rotation axis, and $\nu_{1}=A-B A=2$. On the other side, when $V \gg V_{c 1}$ (i.e. near $V_{c 2}$ ) $\nu_{1}$ assumes its higher value $\nu_{1}^{\max }$ furnished by $A+B A=\nu_{1}^{\max }$; the value of $\nu_{1}^{\max }$ was obtained in Ref. [19] using experimental data of Ref. [22], and is $\nu_{1}^{\max }=2 L\left(V_{c 2}\right) / L_{R}=2.036$. It is seen that the step in $\nu_{1}$ is indeed small. In (2.17), $N^{\prime}$ is a phenomenological coefficient characterizing the rate of growth of $L$ near $V_{c 1}$ and the experimental data show that $N^{\prime}>20$, but do not allow to determine it with precision. Here, we will chose for it the value $N^{\prime}=22$ proposed by Tsubota et al. in Ref. [18.

Expression (2.17) is similar to that proposed in Ref. [19] and it is founded on the microscopic ideas about the nature of the transition, already proposed by Donnelly [5], according to which, for small $V$, the vortex lines are straight lines parallel to the rotation axis, but increasing values of $V$ produce helical perturbations of the vortex lines around their low- $V$ configuration. The situation has been compared by Donnelly to magnetic systems, where the external field $H$ contributes to the orientation of magnetic dipoles, while the temperature $T$ has a disordering effect. Thus, the "tanh" term in (2.17) is analogous to the expression describing magnetization in terms of magnetic field and temperature in a $1 / 2$ spin paramagnetic system. Other expressions, as for instance, Langevin's one for classical paramagnetism, could also be used [18, 28, 29]. This ansatz is similar to that proposed in Ref. [30], to explain the transition from the laminar to the turbulent regime in pure counterflow, and is based on an analogous physical basis: there, the flow was producing the helicoidal excitation waves along 
the vortices pinned to the walls of the container always present in the laminar regime. We recall that in pure counterflow, in containers with circular and square section, there are three different regimes distinguished by two critical counterflow velocities: under the first critical velocity we have the laminar regime where only a few of vortices pinned to the walls of the containers are present, between the two critical velocities a state of low vortex line density (TI regime) is formed, and, at last, above the second critical velocity a state of higher values of $L$ (TII regime) is present. In Ref. [30] the transition from the laminar regime to the turbulent TI regime was explained supposing that small localized arrays of quantized vortices appear when the counterflow velocity reaches the first critical velocity, because Kelvin waves may be propagated in these pinned vortices. Whereas, when the counterflow reaches the second critical velocity, the TI turbulent regime, which is an inhomogeneous and locally polarized state, becomes unstable, with a transition to an homogeneous slightly not isotropic state TII.

In the microscopic model we have commented on, the second critical velocity $V_{c 2}$ is interpreted as the velocity where the helical vortex lines produced in $V_{c 1}$ have reached an amplitude of the order of the average vortex separation and have broken and reconnected, and form a disordered tangle. This explanation is analogous to the one given in Ref. [30] to explain the transition from TI to TII turbulent regimes.

\subsection{Non-stationary solutions of the Vinen's and alternative Vinen's equa- tions}

In this Subsection we study the non-stationary behavior of (2.2) and (2.3). Though the lack of experiments about the evolution of the vortex line density $L$ in this more general case (rotation and counterflow) does not allow us to compare directly our results with experimental data, however we can arrive at some interesting conclusions concerning the difference of behavior.

First of all, we have to state that the analysis below refers to $L$ as dependent variable when the growth of $V$ or of $\Omega$ is very small. Two main situations are considered, in the first one the angular velocity is fixed and the counterflow velocity moves between two consecutive experimental values (see Fig. 11); in the second one the opposite situation is assumed, that is $V$ is fixed and $\Omega$ grows in a small range. This choice is due to the fact that when the ranges of $V$ and $\Omega$ are sufficiently large, the coefficients of the Vinen's equations may be not constant, as showed by Schwarz and Rozen in Ref. [31, because they may depend on the anisotropy and polarization of the tangle.

Denoting with $L_{0}$ the initial value of $L$, the solution of the evolution equation (2.3) is:

$$
\beta \kappa t\left(L_{2}^{A}-L_{1}^{A}\right)=\ln \left|\frac{\left(L-L_{1}^{A}\right)\left(L_{0}-L_{2}^{A}\right)}{\left(L_{0}-L_{1}^{A}\right)\left(L-L_{2}^{A}\right)}\right|,
$$

with $L_{1}^{A}$ and $L_{2}^{A}$ given by (2.13); while the solution of the equation (2.2) can be written as:

$$
\begin{aligned}
-\frac{\beta \kappa}{2} t=\frac{1}{\sqrt{L_{1} L_{2}}} \ln \left|\frac{\sqrt{L}}{\sqrt{L_{0}}}\right| & +\frac{1}{\sqrt{L_{1}}\left(\sqrt{L_{1}}-\sqrt{L_{2}}\right)} \ln \left|\frac{\sqrt{L}-\sqrt{L_{1}}}{\sqrt{L_{0}}-\sqrt{L_{1}}}\right| \\
& +\frac{1}{\sqrt{L_{2}}\left(\sqrt{L_{2}}-\sqrt{L_{1}}\right)} \ln \left|\frac{\sqrt{L}-\sqrt{L_{2}}}{\sqrt{L_{0}}-\sqrt{L_{2}}}\right|,
\end{aligned}
$$

where $L_{0}$ is the initial value of $L$, and $L_{1}$ and $L_{2}$ are expressed by (2.14). 
In order to compare the unsteady solutions (2.19) and (2.20) of (2.3) and (2.2), a value for the coefficient $\beta$ must be chosen. As already said, $\beta$ may depend on the anisotropy and polarization of the tangle, therefore it may have a different value with respect to the one in pure counterflow situation. However, since this dependence is not known in this section, to perform this comparison we choose the value of $\beta$ in pure counterflow, namely $\beta=1 / 2 \pi$.

For $\Omega$ fixed. Now, we choose some values for $V^{2}$ and $\Omega$ in order to plot the solutions of the two models. First of all we consider the case $V^{2}<V_{c 2}^{2}$, and in particular the values $V^{2}=0.0072$ and $\Omega / 2 \pi=0.4$ to which the following values of the stationary solutions correspond:

$$
L_{1}=L_{1}^{A}=5132, \quad L_{2}=4455, \quad L_{2}^{A}=4779 .
$$

For the initial value $L_{0}$ we choose $L_{0}=L_{R}=2 \Omega / \kappa$. Here, all the values for $L, V^{2}$ and $\Omega$ will be expressed in $\mathrm{cm}^{-2}, \mathrm{~cm}^{2} \mathrm{~s}^{-2}$ and $\mathrm{rad} \mathrm{s}^{-1}$, respectively.

From the analysis of Ref. [19] we already know that in this range the stationary solution $L_{1}$ is stable. The same conclusion is reached by looking at the plot of the non-stationary solutions (2.19) and (2.20) of the two models in Fig. 2, Further, we note that the values of $L_{2}$ and $L_{2}^{A}$ are smaller than $L_{1}$ and that the non-stationary solutions approach to the stable stationary one, $L_{1}$, in relatively similar times.

Following the same process as above and setting the same value for $\Omega$ and a value $V^{2}=$ 0.0626 slightly higher than $V_{c 2}^{2}$, we find the following values for the stationary solutions:

$$
L_{1}=L_{1}^{A}=5132, \quad L_{2}=5553, \quad L_{2}^{A}=5476,
$$

and for $L_{0}$ two different values $L_{2_{\mid V^{2}=0.0482}}$ and $L_{2_{\mid V^{2}=0.0482}^{A}}^{A}$ are chosen respectively for the two solutions (2.19) and (2.20) (see Fig. 30). Note that in this case the value of $L_{1}$ is smaller than $L_{2}$ and $L_{2}^{A}$. As we know from previous studies, in this range the stationary solutions $L_{2}$ and $L_{2}^{A}$ for the Vinen's equation and alternative Vinen's one are stable. This is confirmed in Fig. 3, where (2.19) and (2.20) are plotted.

In Fig. 3 we also note a different behavior with respect to that in Fig. 2, in fact, the two non-stationary solutions $L$ approach the corresponding stationary values $L_{2}^{A}$ and $L_{2}$ in rather different times with a ratio of about 1:3, respectively. So, the solution of the alternative Vinen's equation is faster than that of the Vinen's equation.

Furthermore, if we plot the non-stationary solutions for a value of $V^{2}$ much higher than $V_{c 2}^{2}$, we note that the ratio between the temporal scales is yet bigger than the factor 3 . In fact, by setting the same value of $\Omega$ and taking $V^{2}=0.1878$, the corresponding values of the stationary solutions become:

$$
L_{1}=L_{1}^{A}=5132, \quad L_{2}=6910, \quad L_{2}^{A}=7050,
$$

and the graphics of the solutions (2.19) and (2.20) are shown in Fig. 4. As initial data, we have chosen $L_{0}=L_{2_{\mid V^{2}=0.1626}}$ and $L_{0}=L_{\left.\right|_{\mid V^{2}=0.1626} ^{A}}^{A}$ for the Vinen's equation and alternative Vinen's one, respectively. Looking at these unsteady solutions, we note that the solution of the alternative Vinen's equation approaches to $L_{2}^{A}$ in a much shorter time than the other solution requires to approach $L_{2}$, by a ratio of about 1:5.

Note that the time scales in Fig. 3 [100-300 seconds] are much longer than those in Fig. 4 [15-75 seconds]. This is not surprising because Fig. 3 corresponds to a situation which is much closer to the critical velocity $V_{c 2}$ than that corresponding to Fig. 4. Indeed, it is known that 
the dynamics near critical points and phase transitions is much slower than in situations far from them.

For $V$ fixed. In the three situation considered before, the angular velocity is always constant $(\Omega / 2 \pi=0.4)$ whereas the counterflow velocity increase from an initial value $V_{0}$ to a final one $V$. The global behavior is the same when we suppose the opposite situation, that is the counterflow velocity is maintained constant and the angular velocity increases from an initial value $\Omega_{0}$ to a final one $\Omega$. In fact, by choosing the counterflow velocity $V^{2}=0.024<V_{c 2}^{2}$ and increasing $\Omega$ from $\Omega_{0} / 2 \pi=0.95$ to $\Omega / 2 \pi=1$ the following values for the stationary solutions are obtained:

$$
L_{1}=L_{1}^{A}=12831, \quad L_{2}=11345, \quad L_{2}^{A}=12023 .
$$

For $L_{0}$ the value of $L_{1}$ at $\Omega_{0} / 2 \pi=0.95$ is chosen. The plots of the unsteady solutions with the previous values is shown in Fig. 5, from which the same conclusion of Fig. 2 may be reached.

In Fig. 6 the two non-stationary solutions (2.19) and (2.20) are shown when $V^{2}=0.1626>$ $V_{c 2}^{2}$ and $\Omega$ increases from $\Omega_{0} / 2 \pi=0.95$ to $\Omega / 2 \pi=1$. There, by choosing the initial states $L_{0}=L_{2_{\mid \Omega / 2 \pi=0.95}}$ and $L_{0}=L_{2_{\mid \Omega / 2 \pi=0.95}^{A}}^{A}$, the two solutions $L$ approach to the steady states $L_{2}$ and $L_{2}^{A}$ :

$$
L_{1}=L_{1}^{A}=12831, \quad L_{2}=13967, \quad L_{2}^{A}=13767,
$$

in different times, in agreement with the previous situations.

\section{Conclusions}

The possibility of at least two reasonable evolution equations for the vortex line density $L$, namely (1.1) and (1.2), was known since the early days in which Vinen proposed them. However, detailed comparisons for them are very scarce [7, 9]. This was due, in part, to the fact that both of them lead to the same form for the steady state results, namely $L \sim V^{2}$, and that their unsteady solutions are not sufficiently different to reach a definitive conclusion on their relative merit. Here, we have carried out a detailed comparison of an extension of both equations (1.1) and (1.2) to the simultaneous presence of counterflow and rotation. The extension of (1.1) was already studied in Ref. [19]. Here we have studied the analogous extension of (1.2). We have seen that in steady states the solutions of both equations, namely (2.13) and (2.14) have a different form but in the range of available experimental results both of them yield a satisfactory approximate description of the experimental data. However, a deeper comparison of the experimental errors, in (2.15) and (2.16), shows that the description based on the alternative Vinen's equation is slightly better than the one based on the most well-known Vinen's equation.

A new aspect we have explored is the unsteady behavior of the solutions of these equations. Here, both equations exhibit remarkable differences, and we show that the solutions of the alternative Vinen's tend much faster to their steady-state values. In fact, this difference depends on the value of the counterflow velocity. For $V^{2}=0.0626$, slightly higher than the critical velocity $V_{c 2}^{2}$, the time required to reach the steady state solutions is 3 times shorter in the alternative Vinen's equation than in the usual Vinen's equation, whereas for $V^{2}=0.1878$ the difference is still more remarkable, the time scale of the alternative Vinen's equation being 5 times shorter than that for the usual one. Though we lack detailed experimental data on this unsteady behavior, we know that the time required to reach the steady state was less than 10 minutes according to Swanson et al., when the counterflow velocity $V$ is slightly above the 
critical velocity $V_{c 2}$ and it increases between two consecutive experimental values (see pag. 191, Ref. [22]). According to the results of the Fig. 3, the temporal scale of the solution of the usual Vinen's equation is closer to the observations than the temporal scale corresponding to the alternative equation, which tends too fast to the final result. Thus, it seems that the usual equation is preferable on these grounds.

However, it must be stressed that the value of $\beta$ used in our analysis has been $\beta \simeq 0.16$, the value corresponding to pure counterflow, but the value of $\beta$ could depend on the polarity of the tangle, as mentioned below expressions (2.12). The temporal scale of the solution of the generalized usual Vinen's equation could be set equal to the experimental value by setting $\beta=0.11$; instead, to adjust the temporal behaviour of the generalized alternative Vinen's equation a much more radical change in the value of $\beta$ should be made, setting $\beta=0.03$. However, such a drastic reduction of the value of $\beta$ seems at odds with the fact that $A_{1} / \beta$ in the presence of rotation is smaller than in pure counterflow, as mentioned below (2.12). Thus, the dynamical behavior of the usual Vinen's production terms seems more suitable than the modified one. Our work makes also evident the need of more detailed studies of the dependence of $\beta$ - and other coefficients — on the polarization of the tangle.

\section{Acknowledgments}

We acknowledge the support of the Acción Integrada España-Italia (Grant S2800082F HI20040316 of the Spanish Ministry of Science and Technology and grant IT2253 of the Italian MIUR). DJ acknowledges the financial support from the Dirección General de Investigación of the Spanish Ministry of Education under grant BFM 2003-06033 and of the Direcció General de Recerca of the Generalitat of Catalonia, under grant 2005 SGR-00087. MSM and MS acknowledge the financial support from MIUR under grant "PRIN 2005 17439-003" and by "Fondi 60\%" of the University of Palermo. MS acknowledges the "Assegno di ricerca" of the University of Palermo.

\section{References}

[1] W. F. Vinen and J. J. Niemela, J. Low Temp. Phys. 128 (2002) 167

[2] C. F. Barenghi, R. J. Donnelly and W. F. Vinen, Quantized Vortex Dynamics and Superfluid Turbulence, Springer-Verlag Berlin, 2001

[3] W. F. Vinen, Phys. Rev. B 61 (2000) 1410

[4] C. F. Barenghi, J. Phys. Cond. Matter 11 N 40 (1999) 7751

[5] R. J. Donnelly, J. Phys. Cond. Matter 11 N 40 (1999) 7783

[6] W. F. Vinen, Proc. Roy.Soc. London A 240 (1957) 493

[7] W. F. Vinen, Proc. Roy. Soc. London A 243 (1958) 400

[8] S. K. Nemirovskii and W. Fiszdon, Rev. Mod. Phys. 67 N.1 (1995) 37

[9] S. K. Nemirovskii, G. Stamm and W. Fiszdon, Phys. Rev. B 48 (1993) 7338

[10] K. W. Schwartz, Phys. Rev. B 18 (1978) 245 
[11] T. Lipniacki, in Quantized Vortex Dynamics and Superfluid Turbulence, C. F. Barenghi, R. J. Donnelly and W. F. Vinen (eds) 2001, pp. 177

[12] L. D. Landau and E. M. Lifshitz, Statistical Physics, Pergamon Oxford, 1980

[13] D. Jou D, G. Lebon and M. S. Mongiovì, Phys. Rev. B 66 (2002) 224509

[14] D. Kivodites, C. F. Barenghi and D. C. Samuels, Phys. Rev. Lett. 87 (2001) 155301

[15] M. S. Mongiovì and D. Jou, in Condensed Matter: New Research, M. P. Das ed., Nova Science Publishers New York, 2006

[16] A. P. Finne and al., Letters to Nature 424 (2003) 1022

[17] M. Tsubota, T. Araki and C. F. Barenghi, Phys. Rev. Lett. 90 (2003) 205301

[18] M. Tsubota, C. F. Barenghi, T. Araki and A. Mitani, Phys. Rev. B 69 (2004) 134515

[19] D. Jou and M. S. Mongiovì, Phys. Rev. B 69 (2004) 094513

[20] D. Jou and M. S. Mongiovì, Phys. Rev. B 72 (2005) 144517

[21] R. P. Feynman, Chapter 2 in Progress in Low Temperature Physics I, North-Holland Publishing Co., Gorter C J ed. Amsterdam, 1995

[22] C. E. Swanson, C. F. Barenghi and R. J. Donnelly, Phys. Rev. Lett. 50 (1983) 190

[23] E. J. Yarmchuck and W. I. Glaberson, Phys. Rev. Lett. 41 (1978) 564

[24] D. Cheng, M. W. Cromar and R. J. Donnelly, Phys. Rev. Lett. 31 (1973) 433

[25] R. M. Ostermeir and W. I. Glaberson, J. Low Temp. Phys. 21 (1975) 191

[26] M. S. Mongiovì and D. Jou, Phys. Rev. B 72 2005) 104515

[27] W.F. Vinen, Phys. Rev. B 61 (2000) 1410

[28] D. Jou and M. S. Mongiovì, Phys. Rev. B 74 (2006) 054509

[29] D. Jou and M. S. Mongiovì Phys. Lett. A 359 (2006) 183

[30] M. S. Mongiovì and D. Jou, J. Phys: Cond. Matt. 17 (2005) 4423

[31] K. W. Schwarz and J. R. Rozen, Phys Rev. B 44 (1991) 7563

[32] H. E. Hall and W. F. Vinen, Proc. Roy. Soc. London A 238 (1956) 215

[33] R. J. Donnelly, Quantized Vortices in Helium II, Cambridge University Press Cambridge, 1991 


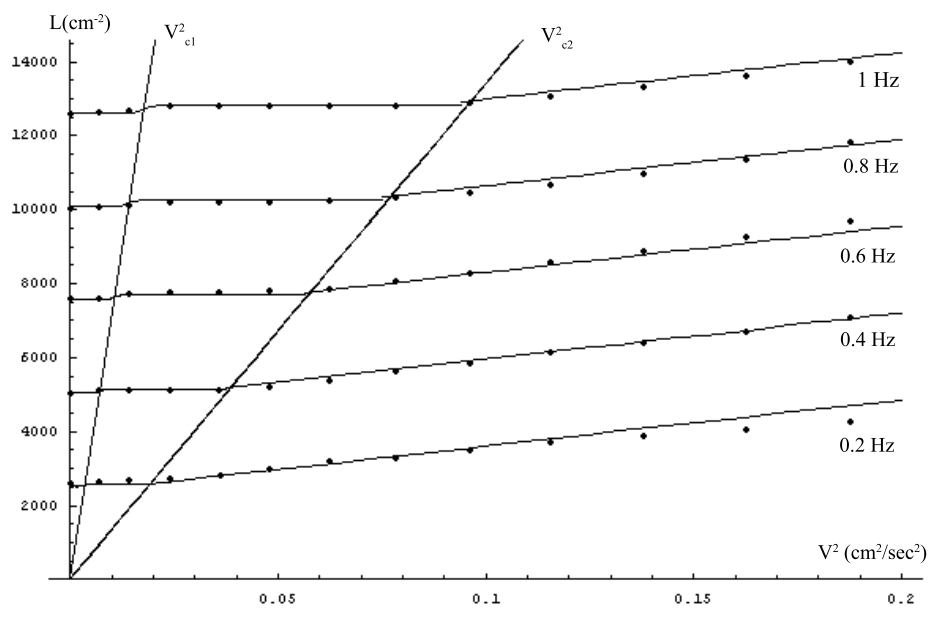

Figure 1: Comparison of the solutions (2.13) (continuous line) with the experimental data by Swanson et al. [22].

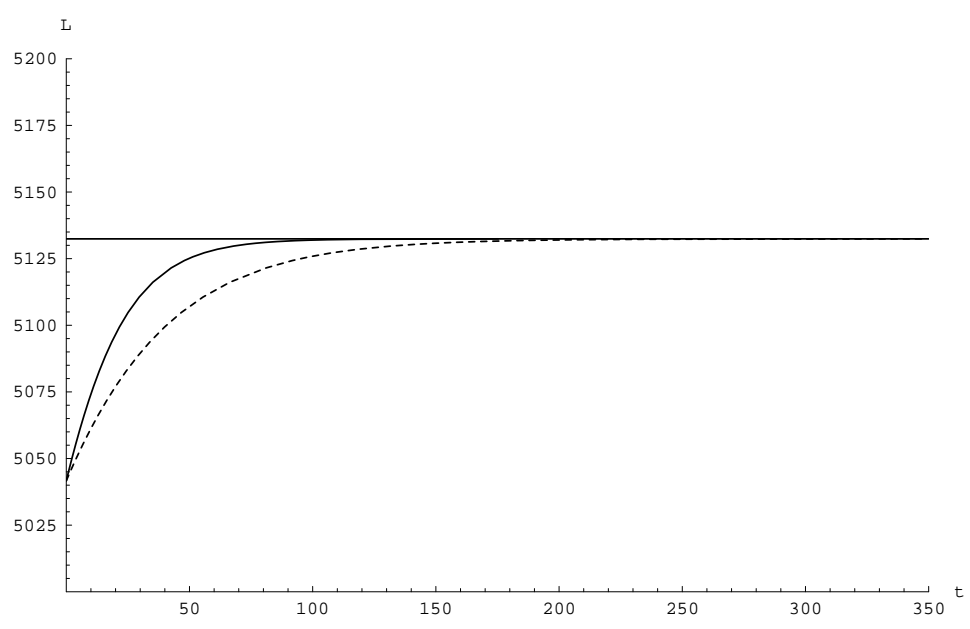

Figure 2: Evolution of the vortex line density $L$ towards its steady state value for the generalizations of the usual Vinen's equation (2.2) [dotted line] and the alternative Vinen's equation (2.3) [continuous line] for $\Omega / 2 \pi=0.4$ and $V^{2}=0.0072$, lower than the critical value $V_{c 2}$. 


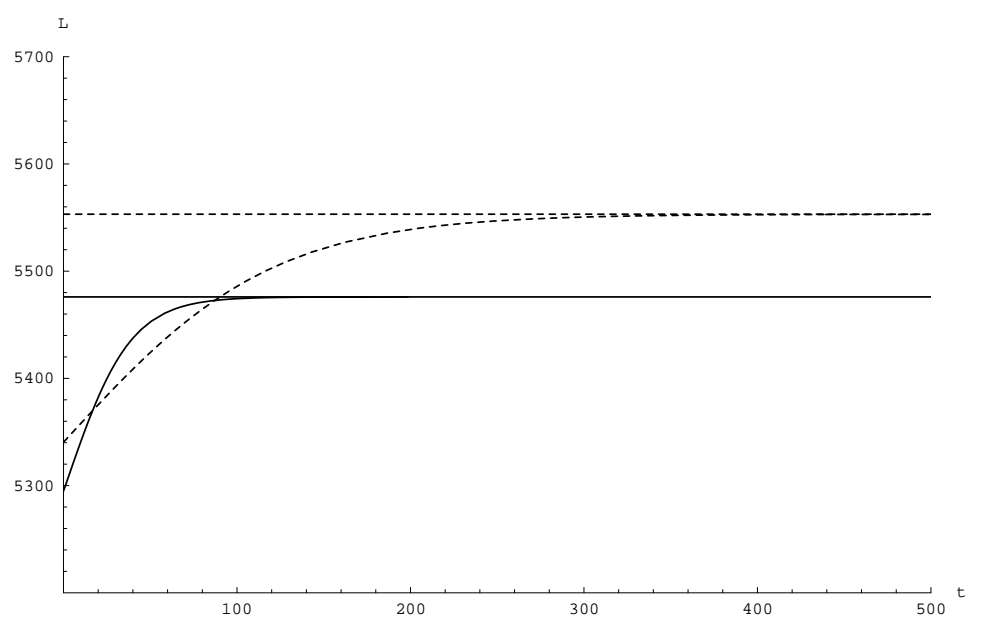

Figure 3: As in Fig. 2, but for $\Omega / 2 \pi=0.4$ and $V^{2}=0.0626$ slightly above the critical value $V_{c 2}$. Note that the steady solutions differ only in a $0.75 \%$, whereas the difference in the time necessary to reach the steady state differs in more than $300 \%$. The values of $L_{0}$ are the unsteady solutions $L_{2}$ and $L_{2}^{A}$ at the same $\Omega$ and $V^{2}=0.0482$.

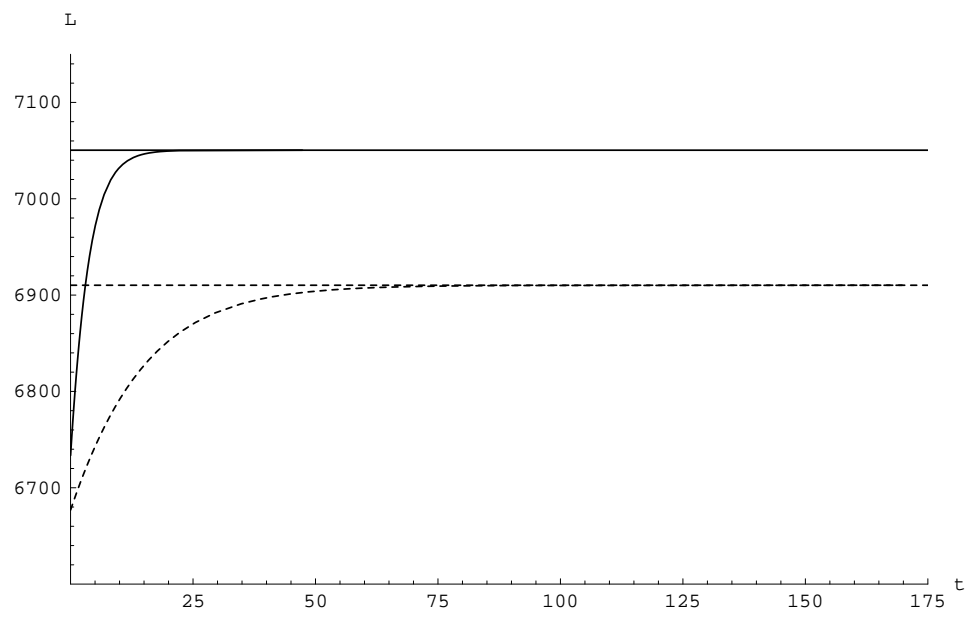

Figure 4: As in Fig. 2, but for $\Omega / 2 \pi=0.4$ and $V^{2}=0.1878$ much higher than the critical value $V_{c 2}$. The times necessary to reach the steady state differ in a $500 \%$ whereas the steady state values differ only in a $12.5 \%$. The values of $L_{0}$ are the unsteady solutions $L_{2}$ and $L_{2}^{A}$ at the same $\Omega$ and $V^{2}=0.1626$. 


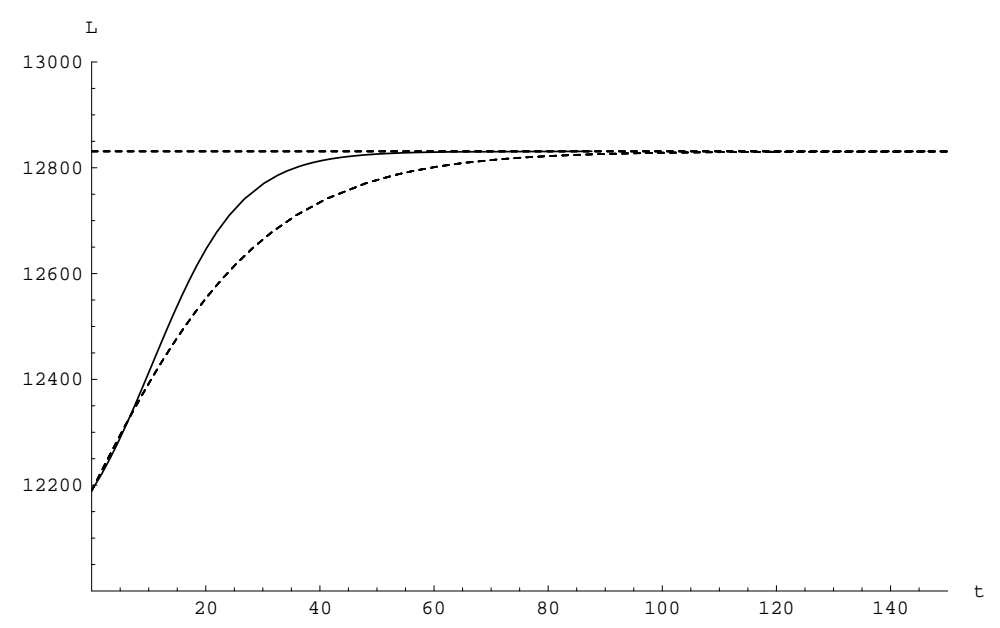

Figure 5: As in Fig. 2, for $\Omega / 2 \pi=1$ and $V^{2}=0.024<V_{c 2}^{2}$, lower than the critical value $V_{c 2}$. Here, the initial data $L_{0}$ is the value of $L_{1}$ at $\Omega / 2 \pi=0.95$ and at the same $V$.

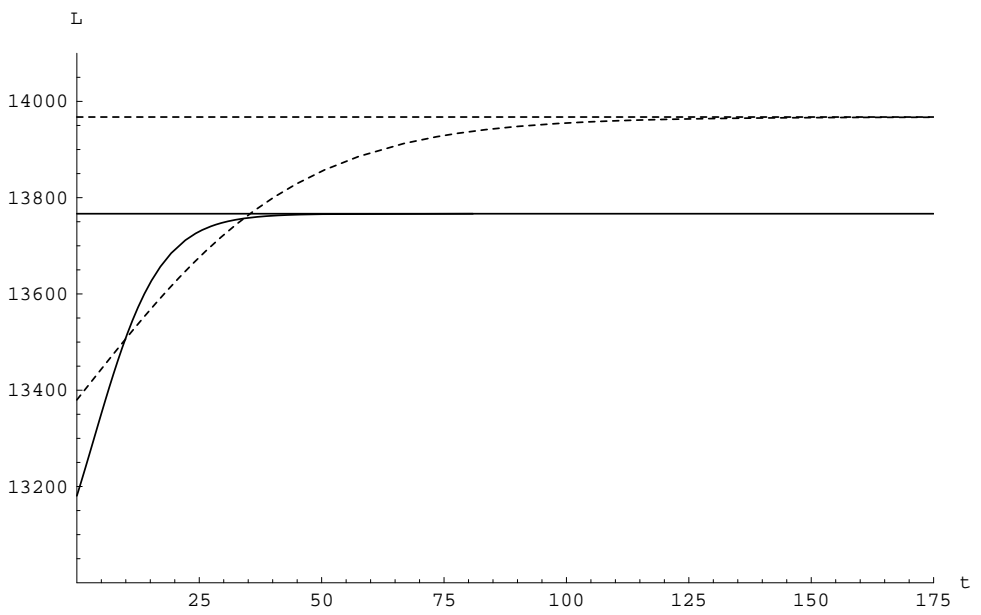

Figure 6: As in Fig. 2, for $\Omega / 2 \pi=1$ and $V^{2}=0.1626$, above the the critical value $V_{c 2}$. The values of $L_{0}$ are the unsteady solutions $L_{2}$ and $L_{2}^{A}$ at the same $V^{2}$ and $\Omega / 2 \pi=0.95$. 